\title{
Accurate Monitoring of Renal Injury State through in Vivo Magnetic Resonance Imaging with Ferric Coordination Polymer Nanodots
}

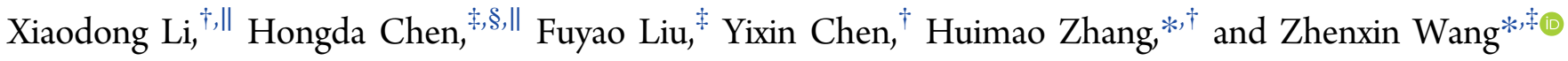 \\ ${ }^{\dagger}$ Department of Radiology, The First Hospital of Jilin University, Changchun 130021, China \\ ${ }^{\ddagger}$ State Key Laboratory of Electroanalytical Chemistry, Changchun Institute of Applied Chemistry, Chinese Academy of Sciences, \\ Changchun 130022, China \\ ${ }^{\S}$ University of Chinese Academy of Sciences, Beijing 100039, China
}

\section{Supporting Information}

ABSTRACT: It is highly challenging to detect the pathophysiology of the diseased kidneys and achieve precise diagnosis because there are few in vivo noninvasive imaging techniques to quantitatively assess kidney dysfunction. This longstanding challenge is normally attributed to the limited molecular contrast agents which can be addressed with renal clearable nanoprobes. In this report, we demonstrate the use of magnetic resonance imaging along with renal clearable ferric coordination polymer nanodots (Fe-CPNDs) for in vivo monitoring the kidney dysfunction effects following drug (daunomycin)-induced kidney injury. After intravenous

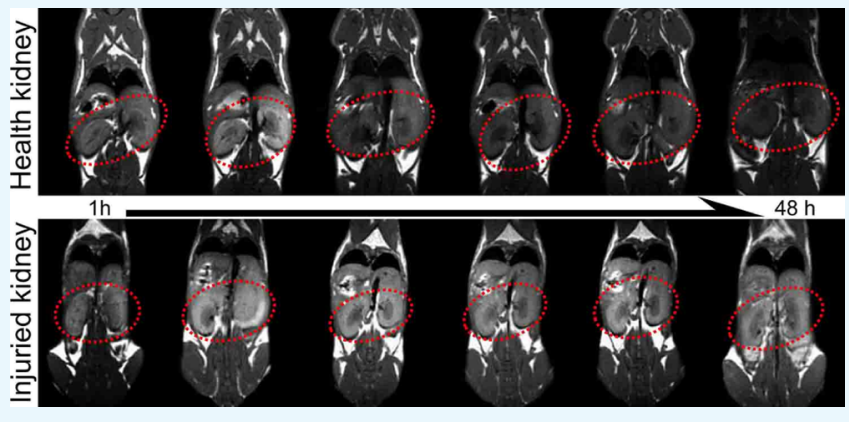
injection of Fe-CPNDs, the change of the MR signal in the kidney can be precisely correlated with local pathological lesion which is demonstrated by renal anatomic details and biochemical examinations of urine and blood. This finding opens the door to the possibility of noninvasively assessing kidney dysfunction and local injuries.

\section{INTRODUCTION}

Kidney injury/dysfunction normally means serious diseases with severe consequences. ${ }^{1-3}$ Intravital molecular imaging techniques are essential tools in the diagnosis and therapy of renal diseases including pyelitis, nephrolithiasis, and tumors. ${ }^{4-10}$ Currently, computed tomography $(\mathrm{CT}),{ }^{4}$ ultrasound imaging, ${ }^{5}$ nuclear imaging, ${ }^{6}$ and magnetic resonance imaging $(\mathrm{MRI})^{7}$ are widely used for both clinical diagnosis of renal diseases and preclinical kidney function studies. Because of the intrinsic insensitivities of $\mathrm{CT} / \mathrm{MRI}$ technique modalities, high dosages of contrast agents (CAs) ( $>60 \mathrm{mg} \mathrm{kg}^{-1}$ body weight) are necessary for evaluation of the kidney function which requires dynamic imaging of kidneys at high temporal resolution and high contrast. ${ }^{11-13}$ For instance, dynamic contrast-enhanced MRI (DCE-MRI) has been proven as a powerful tool in the measurement of the single-kidney glomerular filtration rate through monitoring the kidney clearance kinetics (KCK) of gadolinium (Gd)-based CAs such as Magnevist (Gd-DTPA, DTPA = diethylene triamine pentaacetate) and Dotarem $\left[\mathrm{Gd}(\right.$ DOTA $) \cdot \mathrm{H}_{2} \mathrm{O}$, DOTA = 1,4,7,10-tetraazacyclododecane-1,4,7,10-tetraacetic acid]. Unfortunately, DCE-MRI suffers severely from the nephrotoxicity of Gd-based CAs which are involved in the development of nephrogenic systemic fibrosis in patients with reduced kidney function. ${ }^{14,15}$

Various inorganic/organic gadolinium-free nanoparticles (NPs) with ultrasmall $(<7 \mathrm{~nm})$ hydrodynamic diameters
(HDs) have been arisen as potential CAs for molecular imaging of the kidneys and are receiving increased attention because of the possibility of three-dimensional (3D) images with high spatial resolution and efficient renal clearance. ${ }^{16-21}$ Very recently, Zheng and coauthors have demonstrated that renal clearable gold NPs (AuNPs)-based noninvasive X-ray imaging can be used to precisely discern local pathological lesion in nephropathic kidneys. ${ }^{21}$ In our previous study, we demonstrate that ultrasmall poly(vinylpyrrolidone) (PVP)protected $\mathrm{Fe}^{3+}$-gallic acid (GA) coordination polymer nanodots (Fe-CPNDs) can be used as highly efficient $T_{1}$ CAs with strong MR enhancement and low toxicity. ${ }^{22}$ Unlike commercially available iron oxide CAs (e.g., Feridex I.V.) which is metabolized by the liver, Fe-CPNDs are eliminated from body through renal clearance. Although MRI is nonionizing radiation and can produce noninvasive images with high spatial resolution anatomic details based on soft-tissue contrast, there are few examples on the applications of renal clearable NP-based MRI for studying kidney dysfunction.

Herein, the effect of daunomycin treatment on mice with renal injury has been investigated by renal clearable Fe-CPNDsbased MRI because the Fe-CPNDs exhibit remarkable kidneypositive signal enhancement and increase significantly the

Received: March 19, 2018

Accepted: April 25, 2018

Published: May 4, 2018 


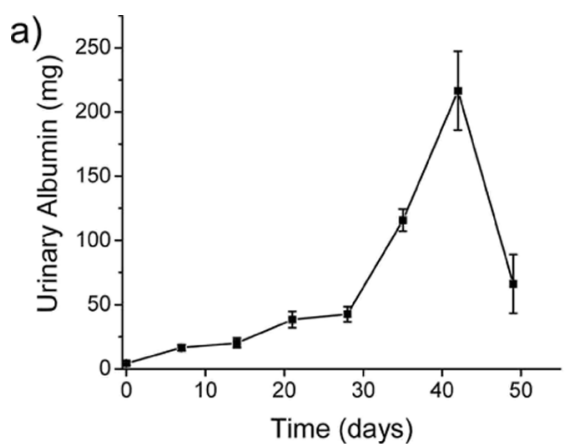

c)

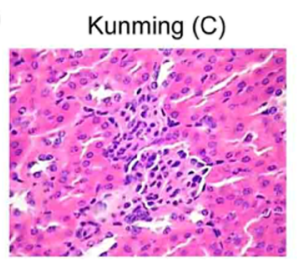

d)

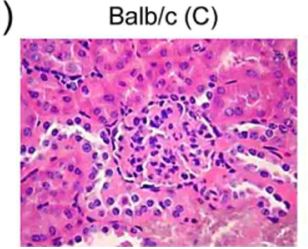

Kunming (+)

Balb/c (+) b)
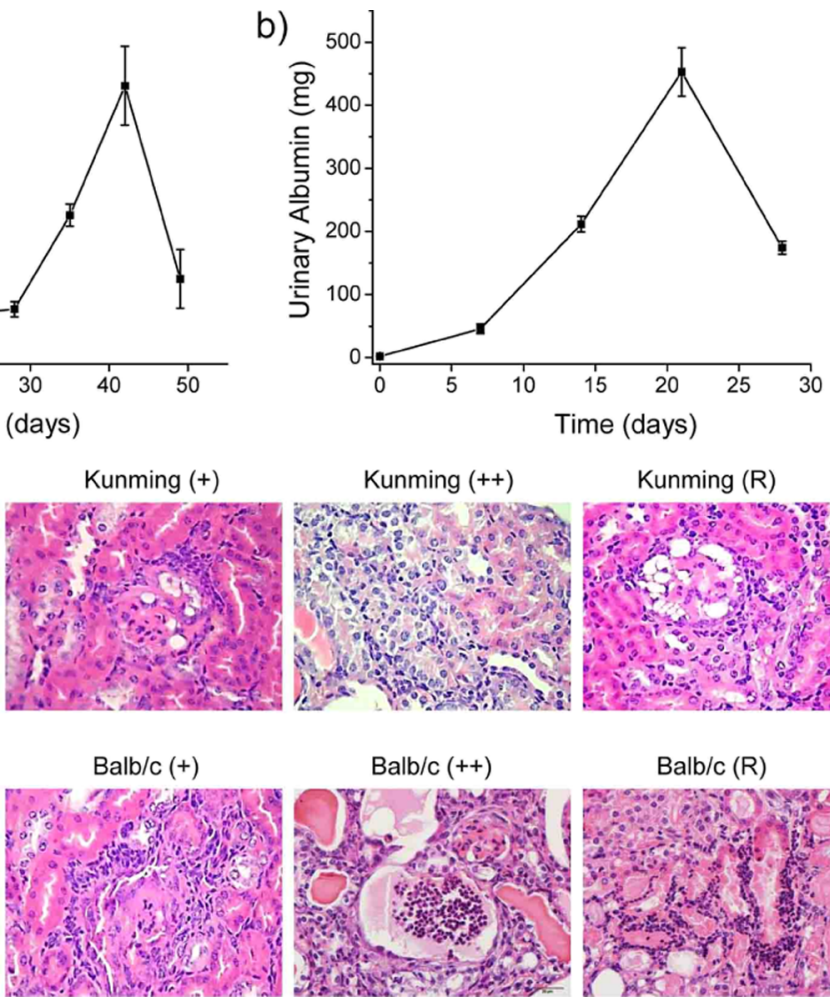

Kunming (++)

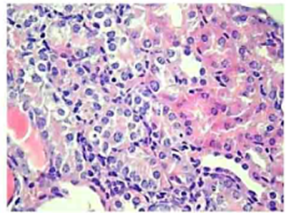

Kunming $(R)$

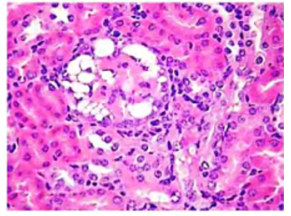

Balb/c (++)

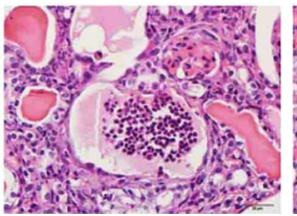

Balb/c (R)

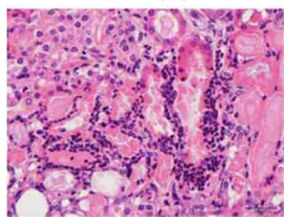

Figure 1. The amount of urinary protein in $1 \mathrm{~mL}$ of (a) Kunming mouse urine and (b) Balb/c mouse urine as a function of time of postinjection daunomycin. Error bars mean standard deviations $(n=5)$. Histological changes in kidneys via staining with H\&E of $(c)$ Kunming mice and $(d)$ $\mathrm{Balb} / \mathrm{c}$ mice. The kidneys of healthy mice are shown as Kunming (C) and (d) Balb/c (C).

imaging-time window. We found that the extent of daunomycin-induced renal injury can be clearly defined by the KCK of Fe-CPNDs.

\section{RESULTS AND DISCUSSION}

Daunomycin-Induced Mouse Kidney Injury Model. A single dose of daunomycin (12 $\mathrm{mg} \mathrm{kg}^{-1}$ body weight) was injected through the tail vein in the Kunming mouse and/or the $\mathrm{Balb} / \mathrm{c}$ mouse to induce different degrees of renal injuries. ${ }^{23-26}$ Two mouse strains are used in this study because different outcomes among mouse strains have been discovered in animal models of renal injuries. ${ }^{27,28}$ After intravenous injection of daunomycin, renal biochemical indicators measurements and histological analysis show that all groups of mice have been induced to different degrees of renal injuries (as shown in Figures 1, S1 and S2). In particular, the degree of kidney injuries exhibits strong time dependence. For instance, the amount of urinary protein in Kunming mouse urine is increased from day 1 postinjection to day 42 postinjection and then gradually decreased from day 42 postinjection to day 49 postinjection. The amount of urinary protein in $\mathrm{Balb} / \mathrm{c}$ mouse urine is increased from day 1 postinjection to day 21 postinjection. The death of daunomycin-treated $\mathrm{Balb} / \mathrm{c}$ mice began to occur at day 22 postinjection. However, if Balb/c mice were treated with continuous methylprednisolone sodium succinate $\left(0.125 \mathrm{mg} \mathrm{kg}^{-1}\right.$ body weight per day, from day 14 to day 21 after injection of daunomycin), the amount of urinary protein could be reduced and the survival rate of mice could be increased. The survival rates of mice in different groups are shown in Figure S3. The result indicates that the daunomycininduced renal injuries in Kunming mice are self-healing, and the daunomycin-induced renal injuries in Balb/c mice can be cured by methylprednisolone sodium succinate treatment. On the basis of amounts of the urinary protein in mice urines, Kunming mice at days 35 and 42 postinjection of daunomycin are defined as mild kidney injury (termed as Kunming $(+)$ ) and severe kidney injury [termed as Kunming $(++)$ ], respectively, while $\mathrm{Balb} / \mathrm{c}$ mice at days 14 and 21 postinjection of daunomycin are defined as mild kidney injury [termed as $\mathrm{Balb} / \mathrm{c}(+)]$ and severe kidney injury [termed as Balb/c $(++)]$, respectively. The self-healing Kunming mice at day 49 postinjection of daunomycin and methylprednisolone sodium succinate-cured $\mathrm{Balb} / \mathrm{c}$ mice at day 28 postinjection of daunomycin are named as Kunming (R) and Balb/c (R), respectively.

The histological analysis [hematoxylin and eosin ( $\mathrm{H} \& \mathrm{E})$ stains] of kidneys with different degrees of injuries is shown in Figure $1 c, d$. Inflammatory cell infiltration is clearly seen in Kunming $(+)$ mice, while inflammatory cell infiltration, glomerular fibrosis, and mesangial cell proliferation are observed in Kunming (++) mice. Balb/c (+) mice have inflammatory cell infiltration and mild hyaline degeneration, while $\mathrm{Balb} / \mathrm{c}(++)$ mice exhibit local degeneration and necrosis of renal tubular epithelial cells, a large number of neutrophils infiltration, a large number of neutrophils in renal tubules, infiltration of inflammatory cells in the interstitium, and acute infection. The symptoms of Kunming (R) and Balb/c (R) are slightly milder than those of Kunming $(+)$ and Balb/c (+). The results suggest that the mouse models with different kidney injuries have been successfully established. In addition, these daunomycin-induced mouse kidney injury models have also confirmed by a blood routine test (as shown in Figure S1) and blood biochemical assays including serum creatinine assay and blood urea nitrogen assay (as shown in Figure S2). According 
to clinical standard of renal injury state, $150 \%$ increase in creatinine is defined as risk, $200 \%$ increase in creatinine is defined as injury, and $300 \%$ increase in creatinine is defined as failure.

In Vivo MRI with Fe-CPND Probes. The Fe-CPNDs were synthesized according to our previously reported strategy. ${ }^{22}$ The Fe-CPNDs can be used as $T_{1}$-weighted MRI CAs with excellent renal clearance ability because the Fe-CPNDs exhibit a low value of $r_{2} / r_{1}$ [i.e., the ratio of transverse relaxivity $\left(r_{2}\right)$ to longitudinal relaxivity $\left(r_{1}\right)$ is ca. 1.9.], good monodispersity, and ultrasmall HD (as shown in Figure S4). In addition, the MRI time window could be significantly improved by the Fe-CPNDs because the Fe-CPNDs exhibit long blood circulation half-lives (HLBC $\geq 1.0 \mathrm{~h}$, as shown in Figure S4d). For testing their accumulation and clearance in kidneys, the Fe-CPNDs at a dose of $2 \mathrm{mg} \cdot \mathrm{kg}^{-1}$ body weight were intravenously injected into the healthy mice and mice with different degrees of kidney injuries. The dose of Fe-CPNDs is determined by our previous study. ${ }^{22}$ After injection of the Fe-CPNDs at this dose, the MR signal enhancement of mouse kidneys can be clearly observed. $T_{1}$-weighted MR images were acquired at preinjection and 1,2 , 4, 6, 24, and 48 h postinjection (as shown in Figures 2, 3, S5 and S6). For all of cases, the MR signals of kidneys are increased from 0 to $2 \mathrm{~h}$ postinjection, while the MR signal decay begins at $2 \mathrm{~h}$ postinjection. This phenomenon suggests that the Fe-CPNDs can effectively accumulate in the kidneys and then be eliminated by kidneys. The maximal enhancement factor of Fe-CPNDs is more than 3, which is equal to that of gadobutrol at a dose of $67 \mathrm{mg} \cdot \mathrm{kg}^{-1}$ body weight (containing the $\mathrm{Gd}$ atoms as high as 12-time $\mathrm{Fe}$ atoms in the injected FeCPNDs), a clinically used $T_{1}$-weighted MRI CA, under the same imaging conditions (as shown in Figure S7). However, the retention of Fe-CPNDs in the kidney is much longer than that of gadobutrol $\left(\mathrm{HLBC}<0.3 \mathrm{~h}^{29}\right)$, which allows us to further quantify the clearance kinetics of the NPs in the kidneys with different degrees of injuries. In particular, the decay rate of the MR signal in the kidney is strongly dependent on the degree of kidney injuries and follows the order Kunming $(\mathrm{C})>$ Kunming $(\mathrm{R})>$ Kunming $(+)>$ Kunming $(++)$ and Balb/c $(\mathrm{C})$ $>\mathrm{Balb} / \mathrm{c}(\mathrm{R})>\mathrm{Balb} / \mathrm{c}(+)>\mathrm{Balb} / \mathrm{c}(++)$. For instance, $\mathrm{MR}$ signals in both Kunming (C) and Balb/c (C) can be recovered to the preinjection levels at $48 \mathrm{~h}$ postinjection. Compared with MR signals in kidneys at preinjection, clearly contrast enhancements (>1.5 times) in Kunming $(++)$ and Balb/c (+ $+)$ can also be observed until $48 \mathrm{~h}$ postinjection. The result indicates that the transport of Fe-CPNDs in the kidney indeed can be noninvasively monitored at high contrast by in vivo MRI. The clearance kinetics of Fe-CPNDs in different kidneys are used to evaluate the renal function because the elimination of Fe-CPND is significantly slowed down in the dysfunctional kidney. The degree of kidney injuries can be semi-quantitatively analyzed by comparing the decay half-lives (DHL) of the timedependent MR density curves derived from kidneys in both the $\mathrm{Balb} / \mathrm{c}$ mouse group and Kunming mouse group (as shown in Figure 3). For instance, DHL ( $>35 \mathrm{~h}$ ) of the mouse with severe kidney injury is much longer than that $(<20 \mathrm{~h})$ of the mouse with mild kidney injury. In addition, such differentiation of renal injury cannot be achieved with gadobutrol-enhanced in vivo MRI (as shown in Figure S7).

The clearance kinetics of the NPs in the kidneys with different degrees of injuries were further confirmed by determination of the Fe element in mouse urine and Prussian blue (PB)-stained kidney tissue. After a single intravenous
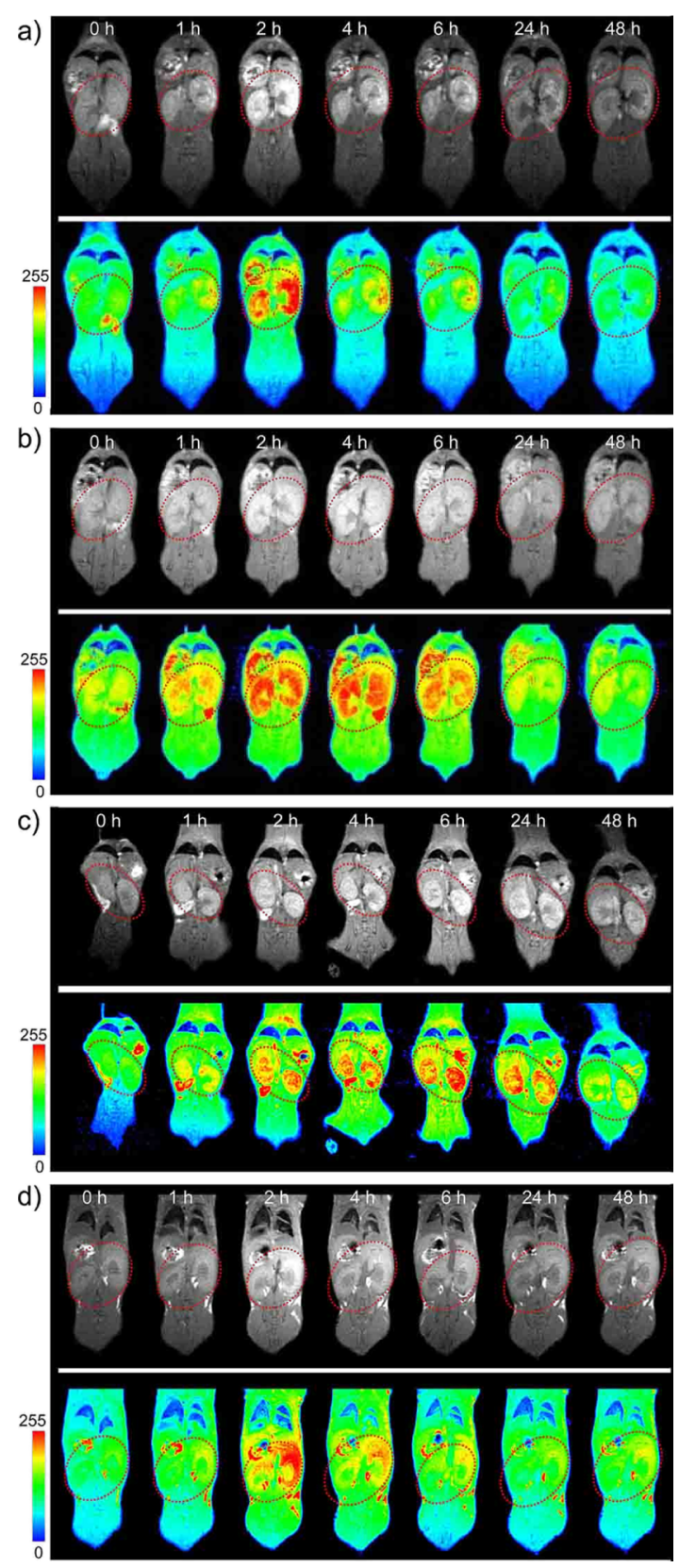

Figure 2. In vivo $T_{1}$-weighted $M R$ images of the kidneys (indicated by red ellipses) with different degrees of injuries [(a) Balb/c (C), (b) Balb/c (+), (c) Balb/c (++), and (d) Balb/c (R)] after the intravenous injection of Fe-CPNDs at different time intervals $(0 \mathrm{~h}$ indicates preinjection). The mice were intravenously injected with Fe-CPNDs at a dose of $2 \mathrm{mg} \cdot \mathrm{kg}^{-1}$ body weight.

injection of $\mathrm{Fe}-\mathrm{CPNDs}$ at a dose of $2 \mathrm{mg} \mathrm{Fe} \mathrm{kg}^{-1}$ body weight, total amounts of the Fe element in the urine of mice at selected time points of postinjection were measured by ICP-MS. As shown in Figure 4, the total amount of $\mathrm{Fe}$ in urine is increasing over time. The half excretion time (HET) of Fe-CPNDs is dependent on the degree of kidney injuries, that is, the mouse with severely injured kidney has a much longer HET than those of mouse with mildly injured kidney and health mouse. PB histochemical staining, which is selective for ferric ions, was performed to detect the iron content in the kidney sections. In this case, Fe-CPNDs can be directly visualized by a blue hue. Figure S8 shows pronounced differences between the injured kidney section and the health kidney section at $24 \mathrm{~h}$ 

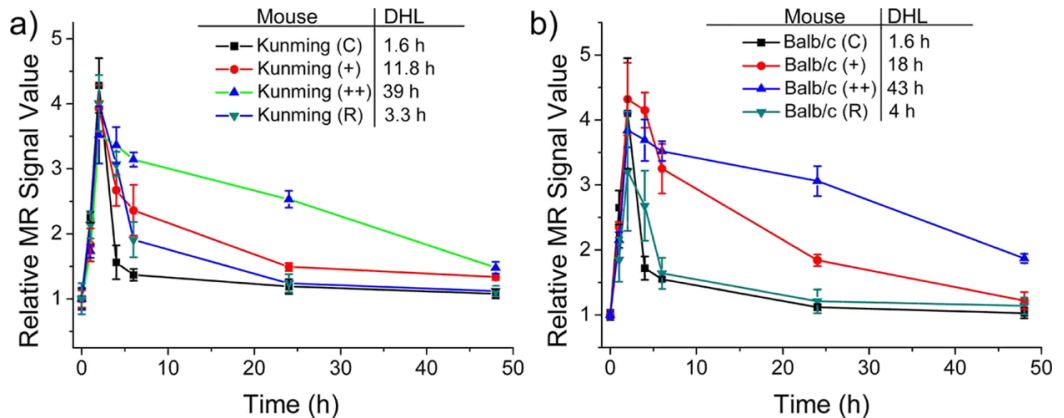

Figure 3. Change of relative MR density as function of postinjection time. The curves derived from kidneys in both (a) Kunming mouse group and (b) Balb/c mouse group. Error bars mean standard derivations $(n=5)$. The MR densities of kidneys at preinjection $(0 \mathrm{~h})$ were defined as 1 . Insets are corresponding DHLs which are counted from $2 \mathrm{~h}$ postinjection (the time point of maximal MR enhancement).

a)

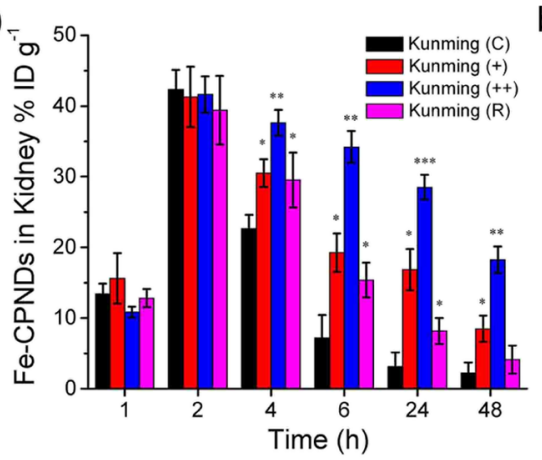

c)

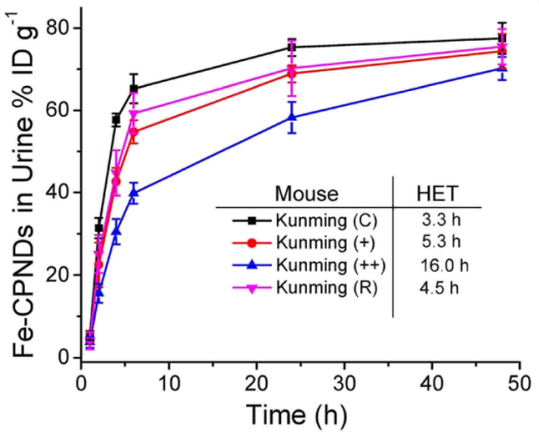

b)

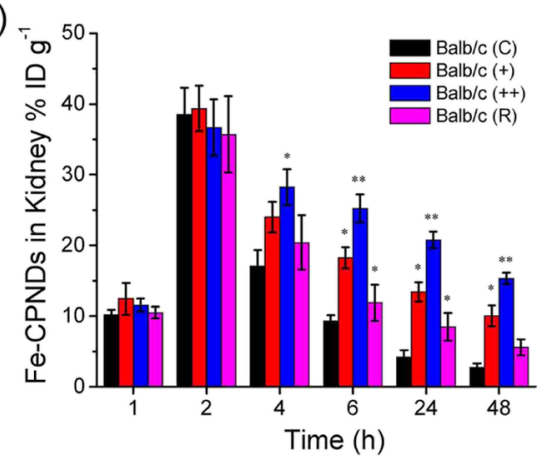

d)

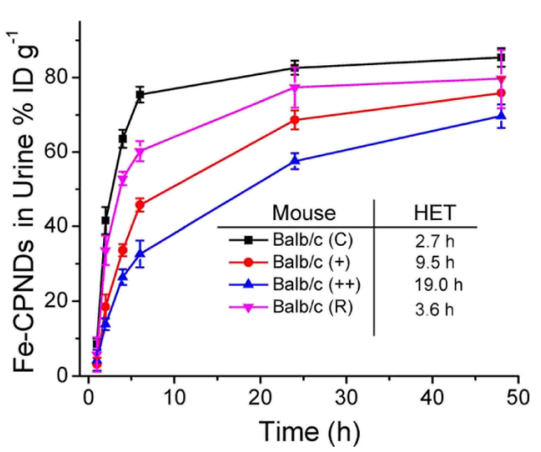

Figure 4. Total amounts of Fe-CPNDs in the mouse kidney $(a, b)$ and urine $(c, d)$ as a function of postinjection times. Error bars mean standard deviations $(n=5)$.

postinjection of Fe-CPNDs. The PB-stained of kidney tissue provides further evidence of the retention of Fe-CPNDs in the injured kidney.

\section{CONCLUSIONS}

In summary, noninvasive dynamic MRI of renal clearable FeCPNDs has proven to be a powerful tool in the quantitative measurement of the degree of kidney injuries because $\mathrm{Fe}$ CPNDs have several desired in vivo behaviors including high $T_{1}$-weighted MR contrast ability, efficient kidney accumulation, and long retention in the blood. Well-defined daunomycininduced kidney injury mouse models were chosen here to demonstrate this new Fe-CPND-based dynamic MRI strategy by proof-of-principle experiments. However, our method shows great promise for noninvasive evaluate kidney function in kidney diseases including chronic kidney disease because it circumvents the nephrotoxicity and the narrow imaging-time window of gadolinium ion-based MRI CAs (e.g., gadobutrol and Magnevist) which are usually clinically used.

\section{EXPERIMENTAL SECTION}

Materials. GA (99\%), iron(III) chloride hexahydrate $\left(\mathrm{FeCl}_{3} \cdot 6 \mathrm{H}_{2} \mathrm{O}\right.$ ), and PVP (molecular weight: $8000 \mathrm{~g} \mathrm{~mol}^{-1}$ ) were obtained from Alfa Aesar (Ward Hill, USA). Creatinine $(\mathrm{Cr})$ assay kit (sarcosine oxidase method), urea nitrogen assay kit, and urine protein assay kit were obtained from Nanjing Jiancheng Bioengineering Institute (Nanjing, China). Daunomycin hydrochloride was obtained from Zhejiang Hisun Pharmaceutical Co., Ltd. (Zhejiang, China). Methylprednisolone sodium succinate was obtained from Guoyao Group Rongsheng Pharmaceutical Co., Ltd. (Henan, China). All other reagents were analytical grade and used without further purification. Milli-Q water $(18.2 \mathrm{M} \Omega \mathrm{cm})$ was used in all experiments.

Preparation of Fe-CPNDs and Mouse Kidney Injury Model. The Fe-CPNDs were synthesized according to our previously reported strategy (see the Supporting Information for details). ${ }^{22}$ 
All animal experiments before initiation were approved by the Institutional Animal Care and Use Committee at Jilin University. All MRI and surgical procedures were processed under intraperitoneal anesthesia using chloral hydrate $(0.25 \mathrm{mg}$. $\mathrm{kg}^{-1}$ body weight). The healthy female Kunming mice and $\mathrm{Balb} / \mathrm{c}$ mice (average mass of about $20 \mathrm{~g}$ ) were purchased from Beijing Vital River Laboratory Animal Technology Co. (Beijing, China). The mice were fed in a clean animal laboratory with a $12 \mathrm{~h} / 12 \mathrm{~h}$ light/dark cycle at $18-22{ }^{\circ} \mathrm{C}$. In all animal experiments, the healthy mice were used as the control group. After adaptive feeding for one week, the mice were injected with a single dose of daunomycin ( $12 \mathrm{mg} \mathrm{kg}^{-1}$ body weight) through the tail vein. The mice were then divided into several groups ( 5 mice per group), and each group was tested at different times to obtain different degrees of kidney injuries. After injection of daunomycin, part of the mice were sacrificed at day 35, day 42, and day 49 (for Kunming mice) and/or at day 14 , day 21 , and day 28 (for Balb/c mice). The kidneys were collected and fixed in $10 \%$ neutral buffered formalin for pathological analysis. The (H\&E-stained or PB-stained) histological sections of kidneys were observed under an optical microscope. For the treatment of the $\mathrm{Balb} / \mathrm{c}$ mice with kidney injury, methylprednisolone sodium succinate was administered intraperitoneally at $0.125 \mathrm{mg} \cdot \mathrm{kg}^{-1}$ body weight every day from day 14 to day 21 after daunomycin injection.

For examination of renal biochemical indicators, the urine and blood were collected at day 35, day 42, and day 49 (for Kunming mice) and at day 14, day 21, and day 28 (for Balb/c mice) postinjection of daunomycin. The urinary protein, serum creatinine, and blood urea nitrogen were measured with commercial assay kits.

In Vivo MRI. At day 35, day 42, and day 49 (for Kunming mice) and at day 14, day 21 , and day 28 (for Balb/c mice) postinjection of daunomycin, $100 \mu \mathrm{L}$ of Fe-CPNDs $\left(2 \mathrm{mg} \cdot \mathrm{kg}^{-1}\right.$ body weight) was injected intravenously into different groups of mice. Mice were anesthetized with isoflurane during scanning. $T_{1}$-weighted MR images were acquired at $1,2,4,6$, 24 , and $48 \mathrm{~h}$ after injection of Fe-CPNDS by the Philips Achieva 3.0T MRI scanner. The following imaging parameters were used including $0.5 \mathrm{~mm}$ (slice thickness), $15 \mathrm{~ms}$ (TE), 358 $\mathrm{ms}$ (TR), $0.8 \mathrm{~mm}$ (in-plane resolution), and $144 \mathrm{~mm} \times 144$ $\mathrm{mm}$ (field of view).

In Vivo Pharmacokinetics and Metabolism. At day 35, day 42, and day 49 (for Kunming mice) and at day 14, day 21, and day 28 (for Balb/c mice) postinjection of daunomycin, 100 $\mu \mathrm{L}$ of Fe-CPNDs (2 $\mathrm{mg} \cdot \mathrm{kg}^{-1}$ body weight) was injected intravenously into different groups of mice. The mice were sacrificed at 1, 2, 4, 6, 24, and $48 \mathrm{~h}$ after injection of Fe-CPNDs. The amounts of Fe nanodots in kidney, blood (collected by the abdominal aortic method), and urine (taken from bladder) of mice were measured by ICP-MS. As a control, the amounts of $\mathrm{Fe}$ in the blood, urine, and kidneys of healthy mice were also determined after injection.

\section{ASSOCIATED CONTENT}

\section{(S) Supporting Information}

The Supporting Information is available free of charge on the ACS Publications website at DOI: 10.1021/acsomega.8b00514.

Preparation of Fe-CPNDs, the blood routine, CR and BUN tests of mice, relaxivities, transmission electron microscopy, $\mathrm{HD}$ and blood circulation in mice of FeCPNDs, in vivo MRI of Kunming mice with different degrees of kidney injuries, MR signal intensities of kidneys, in vivo MRI of the kidneys with different degrees of injuries after intravenous injection of gadobutrol, and PB-stained kidney sections of mice (PDF)

\section{AUTHOR INFORMATION}

\section{Corresponding Authors}

*E-mail: huimaozhanglinda@163.com (H.Z.).

*E-mail: wangzx@ciac.ac.cn. Phone: (+86) 431-85262243. Fax: (+86) 431-85262243 (Z.W.).

\section{ORCID}

Zhenxin Wang: 0000-0002-1908-9848

\section{Author Contributions}

"X.L. and H.C. contributed equally.

\section{Notes}

The authors declare no competing financial interest.

\section{ACKNOWLEDGMENTS}

The authors would like to thank the National Natural Science Foundation of China (grant no. 81571737) for financial support.

\section{REFERENCES}

(1) Nash, K.; Hafeez, A.; Hou, S. Hospital-acquired renal insufficiency. Am. J. Kidney Dis. 2002, 39, 930-936.

(2) Ympa, Y. P.; Sakr, Y.; Reinhart, K.; Vincent, J.-L. Has mortality from acute renal failure decreased? A systematic review of the literature. Am. J. Med. 2005, 118, 827-832.

(3) Chawla, L. S.; Eggers, P. W.; Star, R. A.; Kimmel, P. L. Acute kidney injury and chronic kidney disease as interconnected syndromes. N. Engl. J. Med. 2014, 371, 58-66.

(4) Rob, S.; Bryant, T.; Wilson, I.; Somani, B. K. Ultra-low-dose, lowdose, and standard-dose CT of the kidney, ureters, and bladder: is there a difference? Results from a systematic review of the literature. Clin. Radiol. 2017, 72, 11-15.

(5) McArthur, C.; Baxter, G. M. Current and potential renal applications of contrast-enhanced ultrasound. Clin. Radiol. 2012, 67, 909-922.

(6) Szabo, Z.; Alachkar, N.; Xia, J.; Mathews, W. B.; Rabb, H. Molecular imaging of the kidneys. Semin. Nucl. Med. 2011, 41, 20-28.

(7) Zhang, J. L.; Rusinek, H.; Chandarana, H.; Lee, V. S. Functional MRI of the kidneys. J. Magn. Reson. Imaging 2013, 37, 282-293.

(8) Du, Y.; An, S.; Liu, L.; Li, L.; Zhou, X. J.; Mason, R. P.; Mohan, C. Serial non-invasive monitoring of renal disease following immunemediated injury using near-infrared optical imaging. PLoS One 2012, 7, No. e43941.

(9) Hall, A. M.; Molitoris, B. A. Dynamic multiphoton microscopy: Focusing light on acute kidney injury. Physiology 2014, 29, 334-342.

(10) Hato, T.; Winfree, S.; Dagher, P. C. Intravital imaging of the kidney. Methods 2017, 128, 33-39.

(11) Tofts, P. S.; Cutajar, M.; Mendichovszky, I. A.; Peters, A. M.; Gordon, I. Precise measurement of renal filtration and vascular parameters using a two-compartment model for dynamic contrastenhanced MRI of the kidney gives realistic normal values. Eur. Radiol. 2012, 22, 1320-1330.

(12) Andreucci, M.; Faga, T.; Pisani, A.; Sabbatini, M.; Michael, A. Acute kidney injury by radiographic contrast media: pathogenesis and prevention. BioMed Res. Int. 2014, 2014, 362725.

(13) van Eijs, M. J. M.; van Zuilen, A. D.; de Boer, A.; Froeling, M.; Nguyen, T. Q.; Joles, J. A.; Leiner, T.; Verhaar, M. C. Innovative perspective: gadolinium-free magnetic resonance imaging in long-term follow-up after kidney transplantation. Front. Physiol. 2017, 8, 296. 
(14) Grobner, T. Gadolinium-a specific trigger for the development of nephrogenic fibrosing dermopathy and nephrogenic systemic fibrosis? Nephrol., Dial., Transplant. 2006, 21, 1104-1108.

(15) Marckmann, P.; Skov, L.; Rossen, K.; Dupont, A.; Damholt, M. B.; Heaf, J. G.; Thomsen, H. S. Nephrogenic systemic fibrosis: suspected causative role of gadodiamide used for contrast-enhanced magnetic resonance imaging. J. Am. Soc. Nephrol. 2006, 17, 23592362.

(16) Charlton, J. R.; Beeman, S. C.; Bennett, K. M. NIRI-detectable nanoparticles: the potential role in the diagnosis of and therapy for chronic kidney disease. Adv. Chron. Kidney Dis. 2013, 20, 479-487.

(17) Yu, M.; Liu, J.; Ning, X.; Zheng, J. High-contrast noninvasive imaging of kidney clearance kinetics enabled by renal clearable nanofluorophores. Angew. Chem., Int. Ed. 2015, 54, 15434-15438.

(18) Wang, Y.; Xu, C.; Zhai, J.; Gao, F.; Liu, R.; Gao, L.; Zhao, Y.; Chai, Z.; Gao, X. Label-free Au cluster used for in vivo 2D and 3D computed tomography of murine kidneys. Anal. Chem. 2015, 87, 343345.

(19) Williams, R. M.; Jaimes, E. A.; Heller, D. A. Nanomedicines for kidney diseases. Kidney Int. 2016, 90, 740-745.

(20) Toth, G. B.; Varallyay, C. G.; Horvath, A.; Bashir, M. R; Choyke, P. L.; Daldrup-Link, H. E.; Dosa, E.; Finn, J. P.; Gahramanov, S.; Harisinghani, M.; Macdougall, I.; Neuwelt, A.; Vasanawala, S. S.; Ambady, P.; Barajas, R.; Cetas, J. S.; Ciporen, J.; DeLoughery, T. J.; Doolittle, N. D.; Fu, R.; Grinstead, J.; Guimaraes, A. R.; Hamilton, B. E.; Li, X.; McConnell, H. L.; Muldoon, L. L.; Nesbit, G.; Netto, J. P.; Petterson, D.; Rooney, W. D.; Schwartz, D.; Szidonya, L.; Neuwelt, E. A. Current and potential imaging applications of ferumoxytol for magnetic resonance imaging. Kidney Int. 2017, 92, 47-66.

(21) Xu, J.; Yu, M.; Carter, P.; Hernandez, E.; Dang, A.; Kapur, P.; Hsieh, J.-T.; Zheng, J. In vivo X-ray imaging of transport of renal clearable gold nanoparticles in the kidneys. Angew. Chem., Int. Ed. 2017, 56, 13356-13360.

(22) Liu, F.; He, X.; Chen, H.; Zhang, J.; Zhang, H.; Wang, Z. Gramscale synthesis of coordination polymer nanodots with renal clearance properties for cancer theranostic applications. Nat. Commun. 2015, 6, 8003.

(23) Sternberg, S. S. Cross-striated fibrils and other ultrastructural alterations in glomeruli of rats with daunomycin nephrosis. Lab. Invest. 1970, 23, 39-51.

(24) Luke, D. R.; Vadiei, K.; Hamby, K.; Langston, J. D.; Yau, J. C. Daunorubicin-cyclosporine interaction in the rat. J. Exp. Clin. Cancer Res. 1990, 9, 211-214.

(25) Fujiwara, K.; Shin, M.; Hougaard, D. M.; Larsson, L.-I. Distribution of anticancer antibiotic daunomycin in the rat heart and kidney revealed by immunocytochemistry using monoclonal antibodies. Histochem. Cell Biol. 2007, 127, 69-77.

(26) Shin, M.; Matsunaga, H.; Fujiwara, K. Differences in accumulation of anthracyclines daunorubicin, doxorubicin and epirubicin in rat tissues revealed by immunocytochemistry. Histochem. Cell Biol. 2010, 133, 677-682.

(27) Ma, L.-J.; Fogo, A. B. Model of robust induction of glomerulosclerosis in mice: Importance of genetic background. Kidney Int. 2003, 64, 350-355.

(28) Tewes, S.; Gueler, F.; Chen, R.; Gutberlet, M.; Jang, M.-S.; Meier, M.; Mengel, M.; Hartung, D.; Wacker, F.; Rong, S.; Hueper, K. Functional MRI for characterization of renal perfusion impairment and edema formation due to acute kidney injury in different mouse strains. PLoS One 2017, 12, No. e0173248.

(29) Tombach, B.; Heindel, W. Value of 1.0-M gadolinium chelates: review of preclinical and clinical data on gadobutrol. Eur. Radiol. 2002, 12 , 1550-1556. 